\title{
Bispectral analysis as a probe of quasielastic light scattering intensity fluctuations ${ }^{\text {a) }}$
}

\author{
George D. J. Phillies \\ Department of Chemistry, The University of Michigan, Ann Arbor, Michigan 48109 \\ (Received 4 October 1979; accepted 14 February 1980) \\ The bispectrum $\langle I(0) I(t) I(\tau)\rangle$ of the intensity $I$ of light scattered quasielastically from a fluid system is \\ shown to be a potentially useful tool for studying complex fluids. Bispectra and time cumulants are \\ calculated, in the time domain, for systems with 1,2 , or many diffusing components, treating separately \\ the consequences of homodyne and heterodyne detection at times $0, t$, and $\tau$. The experimentally \\ accessible cumulants of the all-homodyne bispectrum distinguish between systems with exactly two \\ relaxation times and systems with more than two relaxation times. The signal-to-noise ratio in a \\ bispectral measurement is shown to be proportional to $T^{1 / 2}, T$ being the integration time. Clipped scaling \\ of the heterodyne intensity $I(t)$ allows study of the odd powers $\left\langle\left|a_{k}(0) a_{k}^{*}(t) a_{k}(\tau)\right|\right\rangle$ of the density \\ correlations.
}

\section{INTRODUCTION}

In a quasielastic light scattering experiment, one obtains information about a system by illuminating the system with a beam of coherent monochromatic light and studying the temporal behavior of fluctuations in the intensity of the scattered light. The intensity fluctuations are usually characterized by measuring their power spectrum $S_{I}(\omega)$ or their autocorrelation function $G^{(1)}(\tau)$ $=\langle I(t) I(t+\tau)\rangle$. $S_{I}(\omega)$ and $G^{(1)}(\tau)$ are both commonly referred to as the "spectrum" of the scattered light.

It is well known that the higher moments of a stochastic process $X(t)$ often provide a better characterization of the process than the lower moments do. In particular, the triple moment $G^{(2)}(t, \tau)=\langle X(0) X(t) X(\tau)\rangle$ and its double Fourier transform

$$
\begin{aligned}
S_{x}^{(2)}\left(\omega_{1}, \omega_{2}\right)=\iint & d t d \tau\langle X(0) X(t) X(\tau)\rangle \\
& \times \exp \left[-i \omega_{1} t-i \omega_{2} \tau\right],
\end{aligned}
$$

also known as the bispectrum, may contain more information about $X(t)$ than the regular spectrum $S_{x}^{(1)}$ does. Formal aspects of bispectra and polyspectra are treated by Brillinger. ${ }^{1}$

This paper treats some uses of the triple moment ("bispectrum") $G^{(2)}(t, \tau)$ in quasielastic light scattering spectroscopy, including results for scattering from solutions containing one, two, or many noninteracting solute species. With realistic signal-to-noise ratios, $G^{(2)}(t, \tau)$ is shown to give more information about some systems than $G^{(1)}(t)$ does. In particular, in the study of moderately polydisperse systems, measurements of $G^{(1)}(t)$ can readily differentiate between systems characterized by a single decay time and those characterized by a distribution of decay times; however, $G^{(1)}(t)$ can usually not give detailed information about the form of the decay time distribution. In contrast, under the same conditions, $G^{(2)}(t, \tau)$ allows one to distinguish be-

\footnotetext{
a) Acknowledgment is made to the Donors of The Petroleum Research Fund, administered by the American Chemical Society, for partial support of this research. The author is also grateful to The University of Michigan for a Faculty Research Fellowship.
}

tween systems characterized by two decay times and those characterized by three or more decay times. Bispectral analysis may thus be used to validate the "two decay time" models often used in the analysis of nonexponential spectra.

A conventional statistical argument might be inter preted as casting doubt on the utility of bispectral analysis in light scattering spectroscopy. In many fluid systems of experimental interest, the positions and momenta of the component molecules are not correlated over macroscopic distances. The field $E(t)$ of light scattered from bulk equilibrium samples of these fluids is therefore described by a Gaussian random process. Since the higher correlation functions of a Gaussian random process are all determined by its lowest correlation function, one might expect that the bispectrum of light scattered by a large volume of fluid would give only the information already available from the conventional spectrum.

However, real measurements only determine approximate forms for the spectrum and bispectrum. There is no reason to suppose that the partial descriptions of the scattered light given by measured spectra and bispectra will be the same.

While the results are somewhat different, the internal logic of this paper follows that which is used to discuss light scattering spectroscopy. The physical variables which affect the regular spectrum (number of solute species, heterodyne and homodyne detection, signal clipping, and signal-to-noise ratios) are also relevant to bispectral analysis; here each of these is treated in a moderately exhaustive way. We first review some mathematical and chemical results used in the remainder of the paper. In Sec. II, bispectra and their corresponding time cumulants are calculated for experiments using homodyne detection of the light scatter ed from one, two, or many independently diffusing solute species. Section III repeats the calculations of Sec. II for experiments using heterodyne detection and for experiments using a mixture of homodyne and heterodyne detection. In Sec. IV, effects of signal clipping on heterodyne measurements are discussed. Clipping at the average light level changes the symmetry of the bispectrum so that the 
concentration correlation functions which contribute to clipped and unclipped bispectra are not the same. This result also applies to the regular heterodyne spectrum, a point which seems to be overlooked in some discussions. In Sec. V, signal-to-background ratios are calculated; it is also shown that the signal-to-noise ratio in a measurement of the bispectrum is well behaved, depending on the measurement time $T$ as $T^{1 / 2}$. Section VI treats possible designs for a digital bispectral analyzer. Section VII discusses our results, and compares them with other reported applications of bispectral analysis. $^{2-5}$

In the absence of strong absorption or multiple scattering, the amplitude $E_{s}$ of the field of light scattered quasielastically from a fluid system is given by

$$
E_{s}(\mathbf{k}, t)=E_{i} e^{i \omega_{0} t} \alpha(\mathbf{k}, t),
$$

$E_{i}$ and $\omega_{0}$ being the amplitude and frequency of the incident field and $\alpha(\mathbf{k}, t)$ being the (fluctuating) amplitude of the spatial Fourier component of the index of refraction, wave vector $\mathbf{k}$, at time $t$. In an equilibrium solution, $\alpha(k, t)$ is determined by fluctuations in the density $\rho(\mathbf{k}, t)$ and composition $a(\mathbf{k}, t)$, which usually relax on greatly different time scales. At low frequencies, the time-varying envelope of $E_{s}(\mathbf{k}, t)$ is determined entirely by $a(\mathrm{k}, t)$; with an appropriate choice of units,

$$
E_{s}(\mathbf{k}, t)=a(\mathbf{k}, t) e^{i \omega_{0} t} .
$$

For a system containing a single solute component $i, a(\mathbf{k}, t)$ is readily shown to be an Uhlenbeck-Ornstein processs, ${ }^{6}$ in that

$$
\frac{d}{d t}[a(\mathbf{k}, t)]=-\Gamma_{i} a(\mathbf{k}, t)+A_{i}(t),
$$

where $\Gamma_{i}=D_{i} k^{2}, D_{i}$ is the mutual diffusion coefficient of species $i$, and $A_{i}(t)$ is a random source term satisfying $\left\langle A_{i}(t)\right\rangle=0$ and $\left\langle A_{i}(t) A_{i}^{*}\left(t_{+}, \tau\right)\right\rangle=\bar{a}^{2} \delta(\tau)$. One therefore has

$$
a(\mathbf{k}, t)=a(\mathbf{k}, 0) e^{-\Gamma t}+e^{-\Gamma t} \int_{0}^{t} d \zeta A(\zeta) e^{\Gamma \zeta} .
$$

To simplify the notation, we denote $a(\mathrm{k}, t) \equiv a_{t}$.

Two methods of detecting scattered light need be noted. In homodyne detection, one measures the intensity of the scattered light as a function of time, finding

$$
I^{\mathrm{Hom}}(t)=\left|a_{t}\right|^{2} \text {. }
$$

In heterodyne detection, the scattered field is first mixed with a reference field $E_{0} e^{i \omega_{0} t}$ and then detected, so that

$$
I^{\mathrm{Het}}(t)=\left|E_{0}\right|^{2}+2 \operatorname{Re}\left(E_{0}^{*} a_{t}\right)+\left|a_{t}\right|^{2} \text {. }
$$

In a single-detector experiment, the phase of $E_{0}$ is arbitrary. By suitable choice of the origin, one may choose $E_{0}$ to be a real number, so that the cross term in Eq. (4) becomes

$$
2 \operatorname{Re}\left(E_{0}^{*} a_{t}\right)=E_{0}\left(a_{t}+a_{t}^{*}\right) .
$$

It will be necessary to generalize on uses of cumulant (central moment) analysis in light scattering spectroscopy. Koppel ${ }^{7}$ has demonstrated how one may obtain the cumulants of the distribution $A(\Gamma)$ of exponentials in the scattering spectrum of a polydisperse suspension. The spectrum is given by

$$
G^{(1)}(t)=\int d \Gamma A(\Gamma) e^{-\Gamma t}+B
$$

After subtracting the baseline $B$, the cumulants of $A(\Gamma)$ are obtained as the logarithmic derivatives at $t=0$ of $G^{(1)}(t)-B$. Equivalently, the cumulants $K_{n}$ are generated by the power series expansion

$$
G^{(1)}(t)-B=\exp \left(\sum_{n} \frac{K_{n}(-1)^{n} t^{n}}{n !}\right),
$$

which may be generalized for the bispectrum as

$$
C^{(2)}(t, \tau)=\exp \left(\sum_{n \rightarrow m=0}^{\infty} \frac{K_{n m}(-1)^{n+m} t^{n} \tau^{m}}{n ! m !}\right) .
$$

The $K_{n m}$ will be referred to as the time cumulants. Without extraordinary experimental effort, measurements of $G^{(1)}(t)$ only give $K_{0}, K_{1}$, and $K_{2}$. An analogous limit on Eq. (1.11) to terms quadratic in time would allow bispectral determination of $K_{01}, K_{10}, K_{20}, K_{11}$, and $K_{02}$.

By fitting a power series to $G^{(1)}(t)-B$ rather than to $C^{(1)}(t)$ itself, a further implicit use has been made of a cumulant expansion. $\langle I(0) I(t)\rangle$ is the second moment of the stochastic variable $I$; for a two-component system

$$
\langle I(0) I(t)\rangle=\alpha\left\langle I^{2}\right\rangle e^{-\Gamma t}+\langle I\rangle^{2},
$$

and

$$
C^{(1)}(t) \equiv G^{(1)}(t)-B=\langle I(0) I(t)\rangle-\langle I(0)\rangle\langle I(t)\rangle
$$

is the second cumulant of $I$. Cumulant expansions of $I$ are as useful for analyzing bispectra as for analyzing regular spectra, the third cumulant of $I$ being

$$
\begin{aligned}
C^{(2)}(t, \tau) & \equiv\langle I(0) I(t) I(\tau)\rangle_{c} \\
& \equiv\langle[I(0)-\langle I(0)\rangle][I(t)-\langle I(t)\rangle][I(\tau)-\langle I(\tau)\rangle\rangle
\end{aligned}
$$

or

$$
\begin{aligned}
C^{(2)}(t, \tau)= & \langle I(0) I(t) I(\tau)\rangle-\langle I(0)\rangle\langle I(t) I(\tau)\rangle-\langle I(t)\rangle\langle I(0) I(\tau)\rangle \\
& -\langle I(\tau)\rangle\langle I(0) I(t)\rangle+2\langle I(0)\rangle\langle I(t)\rangle\langle I(\tau)\rangle . \quad(1.14
\end{aligned}
$$

Cumulants of the sort defined by Eqs. (1.13) and (1.14) will be referred to as intensity cumulants.

\section{ALL-HOMODYNE BISPECTRA OF SINGLE AND MULTI-SOLUTE SYSTEMS}

In this section, a calculation is made of the bispectrum of light scattered from a solution containing one or more solutes, using a single detector operated in the homodyne mode. For clarity, the calculation is first made for a single-solute (two component) solution and then repeated for a system containing an arbitrary number of solute species. Combining Eqs. (1.14) and $(1.6)$ gives

$$
\begin{aligned}
C^{(2)}(t, \tau)= & \left\langle\left|a_{0}\right|^{2}\left|a_{t}\right|^{2}\left|a_{\tau}\right|^{2}\right\rangle-\left\langle\left|a_{0}\right|^{2}\right\rangle\left\langle\left|a_{t}\right|^{2}\left|a_{\tau}\right|^{2}\right\rangle-\left\langle\left|a_{t}\right|^{2}\right\rangle\left\langle\left|a_{0}\right|^{2}\left|a_{\tau}\right|^{2}\right\rangle-\left\langle\left|a_{\tau}\right|^{2}\right\rangle\left\langle\left|a_{0}\right|^{2}\left|a_{t}\right|^{2}\right\rangle \\
& +2\left\langle\left|a_{0}\right|^{2}\right\rangle\left\langle\left|a_{t}\right|^{2}\right\rangle\left\langle\left|a_{\tau}\right|^{2}\right\rangle,
\end{aligned}
$$


where $t, \tau \geqslant 0$. The double and quadruple moments are ${ }^{6}$

$$
\begin{aligned}
& \left\langle\left|a_{0}\right|^{2}\right\rangle=\bar{a}^{2}, \\
& \left\langle\left|a_{t}\right|^{2}\left|a_{\tau}\right|^{2}\right\rangle=\bar{a}^{4}\left(1+e^{-2 \Gamma_{t}}\right), \\
& \left\langle a_{t} a_{\tau}^{*}\right\rangle=\bar{a}^{2} e^{-\Gamma|t-\tau|} .
\end{aligned}
$$

The sixfold moment $\left\langle\left|a_{0}\right|^{2}\left|a_{t}\right|^{2}\left|a_{\tau}\right|^{2}\right\rangle$ may be obtained from Eq. (1.5) by using the procedures of Uhlenbeck and Ornstein, namely,

$$
\begin{aligned}
\left\langle\left|a_{0}\right|^{2}\left|a_{t}\right|^{2}\left|a_{\tau}\right|^{2}\right\rangle= & \left\langle| a _ { 0 } | ^ { 2 } e ^ { - 2 \Gamma ( t + \tau ) } \left\{\left|a_{0}\right|^{4}+\left|a_{0}\right|^{2} \int_{0}^{\tau} \int_{0}^{\tau} d \zeta_{1} d \zeta_{2} e^{\Gamma\left(\zeta_{1}+\zeta_{2}\right)} A\left(\zeta_{1}\right) A^{*}\left(\zeta_{2}\right)\right.\right. \\
& +2\left|a_{0}\right|^{2} \int_{0}^{t} \int_{0}^{\tau} d \zeta_{1} d \zeta_{2} e^{\Gamma\left(\zeta_{1}+\zeta_{2}\right)} A\left(\zeta_{1}\right) A^{*}\left(\zeta_{2}\right)+\left|a_{0}\right|^{2} \int_{0}^{t} \int_{0}^{t} d \zeta_{1} d \zeta_{2} e^{\Gamma\left(\zeta_{1}+\zeta_{2}\right)} A_{1}\left(\zeta_{1}\right) A_{2}^{*}\left(\zeta_{2}\right) \\
& \left.\left.+\int_{0}^{t} \int_{0}^{t} \int_{0}^{\tau} \int_{0}^{\tau} d \zeta_{1} d \zeta_{2} d \zeta_{3} d \zeta_{4} \exp \left[\Gamma\left(\zeta_{1}+\zeta_{2}+\zeta_{3}+\zeta_{4}\right)\right] A\left(\zeta_{1}\right) A^{*}\left(\zeta_{2}\right) A\left(\zeta_{3}\right) A^{*}\left(\zeta_{4}\right)\right\}\right\rangle .
\end{aligned}
$$

The necessary integrals are

$$
\begin{aligned}
& \int_{0}^{t_{1}} d \zeta_{1} \int_{0}^{t_{2}} d \zeta_{2} e^{\Gamma\left(\zeta_{1}+\zeta_{2}\right)}\left\langle A\left(\zeta_{1}\right) A\left(\zeta_{2}\right)\right\rangle=0 \\
& \int_{0}^{t_{1}} d \zeta_{1} \int_{0}^{t_{2}} d \zeta_{2} e^{\Gamma\left(\zeta_{1}+\zeta_{2}\right)}\left\langle A\left(\zeta_{1}\right) A^{*}\left(\zeta_{2}\right)\right\rangle=\bar{a}^{2}\left\{\exp \left[+2 \Gamma \min \left(t_{1}, t_{2}\right)\right]-1\right\}, \\
& \int_{0}^{t_{1}} \int_{0}^{t_{1}} \int_{0}^{t_{2}} \int_{0}^{t_{2}} d \zeta_{1} d \zeta_{2} d \zeta_{3} d \zeta_{4} \exp \left[\Gamma\left(\zeta_{1}+\zeta_{2}+\zeta_{3}+\zeta_{4}\right)\right]\left\langle A\left(\zeta_{1}\right) A^{*}\left(\zeta_{2}\right) A\left(\zeta_{3}\right) A^{*}\left(\zeta_{4}\right)\right\rangle \\
& \quad=\bar{a}^{4}\left\{\left(e^{-2 \Gamma t_{1}}-1\right)\left(e^{-2 \Gamma t_{2}}-1\right)+[\exp (+2 \Gamma \min (t, \tau))-1]^{2}\right\} \\
& \left\langle\left|a_{0}\right|^{4}\right\rangle=2\left(\bar{a}^{2}\right)^{2} \\
& \left\langle\left|a_{0}\right|^{6}\right\rangle=6\left(\bar{a}^{2}\right)^{3},
\end{aligned}
$$

where $\min (t, \tau)=t, t \leqslant \tau$ and $\min (t, \tau)=\tau, \tau \leqslant t$. The triple moment of the intensities is

$$
G^{(2)}(t, \tau)=\bar{a}^{b}\left[1+e^{-2 \Gamma t}+e^{-2 \Gamma \tau}+e^{-2 \Gamma|t-\tau|}+2 \exp (-2 \Gamma \max (t, \tau))\right],
$$

while the cumulant average is

$$
C^{(2)}(t, \tau)=2 \vec{a}^{\natural} \exp (-2 \Gamma \max (t, \tau)) \text {. }
$$

From Eq. (2.6), it is seen that the triple moment (bispectrum) of the light scattered from a two-component solution is a single exponential of decay constant $\Gamma$. For this system, the bispectrum contains the same information that the conventional spectrum does, that is, values for the decay constant $\Gamma$ and for the root-mean-square amplitude $\bar{a}$.

All cumulants of order greater than two of a Gaussian random process vanish. For scattering from a large volume of solution, $a_{t}$ ought be Gaussian random, so that one expects

$$
\left\langle\left|a_{0}\right|^{2}\left|a_{t}\right|^{2}\left|a_{\tau}\right|^{2}\right\rangle_{c}=0 \text {, }
$$

which is confirmed by Eqs. (2.1) and (2.6). $\left\langle\left|a_{0}\right|^{2}\left|a_{t}\right|^{2}\left|a_{\tau}\right|^{2}\right\rangle_{c}$ differs from $C^{(2)}(t, \tau)$ by terms

$$
\begin{aligned}
& \left\langle\left|a_{0}\right|^{2}\left|a_{t}\right|^{2}\left|a_{\tau}\right|^{2}\right\rangle_{c} \\
& =C^{(2)}(t, \tau)-2\left\langle a_{0} a_{t}^{*}\right\rangle\left\langle a_{0}^{*} a_{t}\left|a_{\tau}\right|^{2}\right\rangle-2\left\langle a_{0} a_{\tau}^{*}\right\rangle\left\langle a_{0}^{*}\left|a_{t}\right|^{2} a_{\tau}\right\rangle-2\left\langle a_{t} a_{\tau}^{*}\right\rangle\left\langle\left|a_{0}\right|^{2} a_{t} a_{\tau}^{*}\right\rangle \\
& \quad+4\left\langle a_{0} a_{t}^{*}\right\rangle\left\langle a_{t} a_{\tau}^{*}\right\rangle\left\langle a_{0}^{*} a_{\tau}\right\rangle+2\left\langle\left|a_{0}\right|^{2}\right\rangle\left|\left\langle a_{t} a_{\tau}^{*}\right\rangle\right|^{2}+2\left\langle\left|a_{t}\right|^{2}\right\rangle\left|\left\langle a_{0} a_{\tau}^{*}\right\rangle\right|^{2}+2\left\langle\left|a_{\tau}\right|^{2}\right\rangle\left|\left\langle a_{0} a_{t}^{*}\right\rangle\right|^{2},
\end{aligned}
$$

the extra factors of 2 arising from the complex conjugate terms. The four amplitude averages are

$$
\begin{aligned}
& \left\langle a_{0}^{*} a_{t}\left|a_{\tau}\right|^{2}\right\rangle=\bar{a}^{4}\left(e^{-\Gamma t}+\exp \{-\Gamma[t+2 \tau-2 \min (t, \tau)]\}\right), \\
& \left\langle a_{0}^{*}\left|a_{t}\right|^{2} a_{\tau}\right\rangle=\bar{a}^{4}\left(e^{-\Gamma \tau}+\exp \{-\Gamma[2 t+\tau-2 \min (t, \tau)]\}\right), \\
& \left\langle\left|a_{0}\right|^{2} a_{t} a_{\tau}^{*}\right\rangle=\bar{a}^{4}\left(e^{-\Gamma|t-\tau|}+e^{-\Gamma(t+\tau)}\right),
\end{aligned}
$$

which may be combined to yield Eq. (2.7). Equations (2.6) and (2.7) emphasize the difference between the cumulants of the intensity and those of the amplitude, the former containing cross terms which cancel out in the latter.

An extension of the above calculations yields the all-homodyne bispectrum of an $N$-component system. The amplitude $a(\mathbf{k}, t)$ must be replaced with a sum of amplitudes $\sum_{i=1}^{N} a_{i}(\mathrm{k}, t) \equiv \sum_{i=1}^{N} a_{i t}$. Each $a_{i t}$ is separately given by an Uhlenbeck-Ornstein process with decay constant $\Gamma_{i}=D_{i} k^{2}$ and root-mean-square amplitude $\left\langle\left|a_{i}\right|^{2}\right\rangle^{1 / 2}=\bar{a}_{i} \cdot \quad$ For independently diffusing solute components, $\left\langle a_{i t} a_{j \tau}^{*}\right\rangle \propto \delta_{i j}$ and $\left\langle A_{i}(t) A_{j}^{*}(t)\right\rangle=\bar{a}_{i}^{2} \delta_{i j}$. The triple moment of the intensities is 


$$
G^{(2)}(t, \tau)=\sum_{\substack{i, j, l_{i, m} \\ p, q=1}}^{N}\left\langle a_{i 0} a_{j 0}^{*} a_{3 t} a_{m t}^{*} a_{p \tau} a_{\sigma \tau}^{*}\right\rangle,
$$

the cumulant average following from $\mathrm{Eq}$. (1.14). Equation (2.10) is evaluated by considering all terms in which no subset of the six indices containing an odd number of elements has all indices in the subset equal to each other and not equal to any index not in the subset; i.e., one needs only terms in which all indices are pair, four, or sixwise equal. From Eqs. (2.2)-(2.4) and (2.9),

$$
\begin{aligned}
G^{(2)}(t, \tau)= & \sum_{i=1}^{N} \bar{a}_{i}^{6}\left\{1+e^{-2 \Gamma_{i} t}+e^{-2 \Gamma_{i \tau}}+e^{-2 \Gamma_{i}|t-\tau|}+2 \exp \left[-2 \Gamma_{i} \max (t, \tau)\right]\right\} \\
& +\sum_{\substack{i, j \neq 1 \\
i \neq j}}^{N}\left\{\overline { a } _ { i } ^ { t } \overline { a } _ { j } ^ { 2 } \left[3+2 e^{-\left(\Gamma_{i}+\Gamma_{j}\right) t}+2 e^{-\left(\Gamma_{i}+\Gamma_{j}\right) \tau}+2 \exp \left[-\left(\Gamma_{i}+\Gamma_{j}\right)|t-\tau|\right]+\exp \left[-\Gamma_{i}(t+\tau)-\Gamma_{j}|t-\tau|\right]\right.\right. \\
& \left.\left.+\exp \left[-\Gamma_{i}(2 t+\tau-2 \min (t, \tau))-\Gamma_{j} \tau\right]+\exp \left[-\Gamma_{i}(2 \tau+t-2 \min (t, \tau))-\Gamma_{j} t\right]+e^{-2 \Gamma_{i} t}+e^{-2 \Gamma_{i} \tau}+e^{-2 \Gamma_{i} \mid t-\tau i}\right]\right\} \\
& +\sum_{\substack{i, j, i=1 \\
i \neq j \neq i \neq i}}^{N}\left\{\bar{a}_{i}^{2} \bar{a}_{j}^{2} \bar{a}_{i}^{2}\left[1+2 \exp \left(-\Gamma_{i} t-\Gamma_{j} \tau-\Gamma_{i}|t-\tau|\right)+e^{-\left(\Gamma_{i}+\Gamma_{j}\right) t}+e^{-\left(\Gamma_{i}+\Gamma_{j}\right) \tau}+\exp \left(-\left(\Gamma_{i}+\Gamma_{j}\right)|t-\tau|\right)\right]\right\}
\end{aligned}
$$

the corresponding intensity cumulant being

$$
\begin{aligned}
C^{(2)}(t, \tau)=2 & \left(\sum_{i} \bar{a}_{i}^{6} \exp \left[2 \Gamma_{i} \max (t, \tau)\right]+\sum_{\substack{i, j \\
i \neq j}} \bar{a}_{i}^{4} \bar{a}_{j}^{2}\left\{\exp \left(-\Gamma_{i}(t+\tau)-\Gamma_{j}|t-\tau|\right)\right.\right. \\
& \left.+\exp \left[-\Gamma_{i}(2 t+\tau-2 \min (t, \tau))-\Gamma_{j} \tau\right]+\exp \left[-\Gamma_{i}(t+2 \tau-2 \min (t, \tau))-\Gamma_{j} t\right]\right\} \\
& \left.+\sum_{i \neq j \neq k \neq i} \bar{a}_{i}^{2} \bar{a}_{j}^{2} \bar{a}_{k}^{2} \exp \left[-\Gamma_{i} t-\Gamma_{j} \tau-\Gamma_{k}|t-\tau|\right]\right) .
\end{aligned}
$$

A direct calculation confirms that the last sum vanishes if only two solute species are present. $C^{(2)}(t, \tau)$ is again substantially simpler than $G^{(2)}(t, \tau)$.

As in the regular spectrum, trying to obtain the $\bar{a}_{i}$ and $\Gamma_{i}$ from a direct multiexponential fit of Eq. (2.12) ought not be effective. In this section, the information yielded by fitting $C^{(2)}(t, \tau)$ to the time cumulants $K_{n m}$ will be calculated, limiting consideration to terms quadratic in time.

To simplify terms, we may without loss of generality take $0<t<\tau$. For a solution containing a single solute, calculation of the cumulants from Eq. (2.6) shows

$$
\begin{aligned}
& K_{10}=K_{20}=K_{11}=K_{02}=0, \\
& K_{01}=2 \Gamma .
\end{aligned}
$$

Because the triple sum in Eq. (2.12) only appears if three or more solute species are present, it is useful to give separate forms for $N=2$ and $N \geq 3$. For $N=2$ the corresponding cumulants are

$$
\begin{aligned}
& K_{10}=0, \\
& K_{01}=2 \frac{\Gamma_{1} \bar{a}_{1}^{2}+\Gamma_{2} \bar{a}_{2}^{2}}{\bar{a}_{1}^{2}+\bar{a}_{2}^{2}}, \\
& K_{02}=K_{20}=-2 K_{11}=\frac{2 \bar{a}_{1}^{2} \bar{a}_{2}^{2}\left(\Gamma_{1}-\Gamma_{2}\right)^{2}}{\left(\bar{a}_{1}^{2}+\bar{a}_{2}^{2}\right)^{2}} .
\end{aligned}
$$

Finally, for $N \geqslant 3$ the cumulants are

$$
\begin{aligned}
& K_{10}=0, \\
& K_{01}=2 \sum_{i} \bar{a}_{i}^{2} \Gamma_{i} / \sum_{m} \bar{a}_{m}^{2},
\end{aligned}
$$

$$
\begin{aligned}
K_{02}= & K_{20}=\sum_{i, j} \bar{a}_{i}^{2} \bar{a}_{j}^{2}\left(\Gamma_{i}-\Gamma_{j}\right)^{2} /\left(\sum_{m} \bar{a}_{m}^{2}\right)^{2} \\
K_{11}= & \left(-\sum_{i, j} \bar{a}_{i}^{4} \bar{a}_{j}^{2}\left(\Gamma_{i}-\Gamma_{j}\right)^{2}\right. \\
& \left.-\sum_{i \neq j \neq z_{k} \neq i} \bar{a}_{i}^{2} \bar{a}_{j}^{2} \bar{a}_{k}^{2}\left(\Gamma_{j}-\Gamma_{i}\right)\left(\Gamma_{j}+\Gamma_{k}\right)\right) /\left(\sum_{m} \bar{a}_{m}^{2}\right)^{3}
\end{aligned}
$$

In every case, $K_{10}$ is zero; near $t=\tau=0, C^{(2)}(t, \tau)$ does not have a linear dependence on $t$. Similarly, in every case $K_{01}$ gives the intensity-weighted average diffusion coefficient $2 \bar{\Gamma}$. The second order cumulants give information about the range of decay times observed in the scattered light. $K_{02}$ is equal to $K_{2}$, the second cumulant of the regular spectrum; $K_{20}$ and $K_{2}$ both estimate the mean-square range of decay times in the system. The most interesting result is for $K_{20}$ and $K_{11}$. In a one exponential system, $K_{20}$ and $K_{11}$ are zero. In a two exponential system $K_{20}=-2 K_{11}$, but in a system with three or more decay times, $K_{20}$ and $K_{11}$ are not related by a simple constant. That is, by using bispectral anal$y$ sis to determine $K_{11}$ and $K_{20}$, one can determine whether a system has exactly two relaxation times or whether that system has more than two relaxation times.

\section{HETERODYNE AND MIXED BISPECTRA}

In conventional light scattering spectroscopy, homodyne and heterodyne detection give the same information about large equilibrium systems. As long as the scattering volume can be broken into many physically independent subvolumes (a definition which excludes velocimetry measurements in which the velocity of individual 
scatterers is correlated across the entire scattering volume), the central limit theorem guarantees that the spectra $C^{(1)}(t)$ and $C_{\text {Hot }}^{(1)}(t)$ measured by homodyne and heterodyne detection, respectively, are related by

$$
C^{(1)}(t)=\left|C_{\mathrm{Het}}^{(1)}(t)\right|^{2},
$$

so in conventional light scattering spectroscopy homodyne and heterodyne detection give equivalent results.

In this section, the usefulness of heterodyne detection in bispectral analysis is considered. Bispectra are calculated assuming heterodyne detection of the light scattered from solutions containing one or more diffusing solute components. In contrast to the regular spectrum, the homodyne and heterodyne bispectra are not equivalent, the heterodyne bispectra yielding little more information than can be obtained from the regular homodyme spectrum. It is also possible to measure mixed bi spectra, in which a given coherence area of the scattered field is observed both by a homodyne detector and by a heterodyne detector; the mixed bispectra are shown to be no more useful than the bispectrum obtained using only heterodyne detection.

For a solution containing one solute component, the heterodyne bispectrum is

$$
\begin{aligned}
C_{\mathrm{Hot}}^{(2)}(t, \tau)= & \left\langle\left(\left|E_{0}\right|^{2}+2 E_{0} a_{0 R}+\left|a_{0}\right|^{2}-\bar{I}_{0}\right)\right. \\
& \times\left(\left|E_{t}\right|^{2}+2 E_{t} a_{t R}+\left|a_{t}\right|^{2}-\bar{I}_{t}\right) \\
& \left.\times\left(\left|E_{\tau}\right|^{2}+2 E_{\tau} a_{\tau R}+\left|a_{\tau}\right|^{2}-\bar{I}_{\tau}\right)\right\rangle,
\end{aligned}
$$

where $a_{t R}$ and $I_{t}$ are the real part of $a_{t}$ and the average intensity at time $t$, respectively, the average heterodyne intensity being $I_{0}=\left|E_{0}\right|^{2}+\left\langle\left|a_{0}\right|^{2}\right\rangle$. Recalling that the average of an odd power of $a_{0}$ vanishes, the procedures of the previous section give for a one-solute solution

$$
\begin{gathered}
C_{\mathrm{Het}}^{(2)}(t, \tau)=2 E^{2} \bar{a}^{4}\left(e^{-\Gamma(t+\tau)}+e^{-\Gamma \tau}+e^{-\Gamma(2 \tau-t)}\right) \\
+2 \bar{a}^{6} e^{-2 \Gamma \tau},
\end{gathered}
$$

where $0<t<\tau$. All terms of higher order in $E$ vanish in the cumulant average; the $\bar{a}^{6}$ term is exactly analogous to the homodyne background term in a regular heterodyne spectrum. If we assume $E^{2} \gg \bar{a}^{2}$, the cumulants corresponding to $\mathrm{Eq}$. (3.4) are

$$
\begin{aligned}
& K_{10}=0, \\
& K_{01}=\frac{1}{3} \Gamma, \\
& K_{20}=-2 K_{11}=\frac{2}{3} \Gamma^{2}, \\
& K_{02}=\frac{2}{9} \Gamma^{2} .
\end{aligned}
$$

For $N$ solute species, the all-heterodyne bispectrum is

$$
C_{\mathrm{Hot}}^{(2)}(t, \tau)=2 E^{2} \sum_{i, j} \bar{a}_{i}^{2} \bar{a}_{j}^{2}\left[\exp \left(-\Gamma_{i} t-\Gamma_{j} \tau\right)+\exp \left(-\Gamma_{i} t-\Gamma_{j}|t-\tau|\right)+\exp \left(-\Gamma_{i} \tau-\Gamma_{j}|t-\tau|\right)\right]+C_{\mathrm{Hom}}^{(2)}(t, \tau)
$$

For $E^{2} \gg \bar{a}^{2}$, the cumulants are

$$
\begin{aligned}
& K_{10}=0, \\
& K_{01}=4 \sum_{j} \Gamma_{j} \bar{a}_{j}^{2} /\left(3 \sum_{i} \bar{a}_{i}^{2}\right), \\
& K_{20}=-2 K_{11}=2 \sum_{i, j} \bar{a}_{i}^{2} \bar{a}_{j}^{2}\left(2 \Gamma_{i}^{2}-\Gamma_{i} \Gamma_{j}\right) /\left(3 \sum_{i, j} \bar{a}_{i}^{2} \bar{a}_{j}^{2}\right), \\
& K_{02}=\frac{2}{3} \sum_{i, j} \bar{a}_{i}^{2} \bar{a}_{j}^{2}\left(\left(\Gamma_{i}-\Gamma_{j}\right)^{2}+\frac{\Gamma_{i} \Gamma_{i}}{3}\right) / \sum_{i, j} \bar{a}_{i}^{2} \bar{a}_{j}^{2} .
\end{aligned}
$$

The cumulants satisfy $K_{20}=-2 K_{11}$ for all $N$, so that the heterodyne bispectrum does not (through terms quadratic in time) readily distinguish between two and three component systems, though the difference between $K_{20}$ and $K_{02}$ may be useful in some cases.

It is also in principle possible to measure mixed bispectra of forms such as $\left\langle I^{\mathrm{Hom}}(0) I^{\mathrm{Het}}(t) I^{\mathrm{Hom}}(\tau)\right\rangle$. Experimentally, a bispectrum of this form could be obtained by isolating a single coherence area of the scattered field, dividing the scattered light between two detectors with a beam splitter and using a reference beam to operate one detector in the heterodyne mode. The mixed bispectra are found by cross correlating the detector intensities.

The choice of detector may be made independently at each of three times, so six possible mixed spectra exist. Spectra using heterodyne detection at one time are not interesting. These spectra can be written

$$
\begin{aligned}
C_{M M}^{(2)}(t, \tau)= & \left\langle\left(2 E_{1}^{\prime} a_{1 R}+\left|a_{1}\right|^{2}-\bar{a}^{2}\right)\right. \\
& \left.\times\left(\left|a_{2}\right|^{2}-\bar{a}^{2}\right)\left(\left|a_{3}\right|^{2}-\bar{a}^{2}\right)\right\rangle .
\end{aligned}
$$

Only even powers of $a_{i}$ have nonzero averages, so all terms of $C_{\mu M}^{(2)}$ involving $E_{1} a_{1 R}$ average to zero. Ignoring these terms,

$$
C_{M M}^{(2)}(t, \tau) \equiv C^{(2)}(t, \tau),
$$

but $C^{(2)}(t, \tau)$ can be obtained directly.

Spectra using heterodyne detection at two times are marginally more useful. Using the subscript $M i$ to denote homodyne detection at time $i$,

$$
\begin{aligned}
& C_{M 0}^{(2)}(t, \tau)=\left\langle I^{\mathrm{Hom}}(0) I^{\mathrm{Het}}(t) I^{\mathrm{Het}}(\tau)\right\rangle_{c}=2 E^{2} \sum_{i j} \bar{a}_{i}^{2} \bar{a}_{j}^{2} \exp \left(-\Gamma_{i} t-\Gamma_{j} \tau\right)+C^{(2)}(t, \tau) \\
& C_{M t}^{(2)}(t, \tau) \equiv\left\langle I^{\mathrm{Het}}(0) I^{\mathrm{Hom}}(t) I^{\mathrm{Het}}(\tau)\right\rangle_{c}=2 E^{2} \sum_{i j} \bar{a}_{i}^{2} \bar{a}_{j}^{2} \exp \left(-\Gamma_{i} t-\Gamma_{j}|t-\tau|\right)+C^{(2)}(t, \tau) \\
& C_{M \tau}^{(2)}(t, \tau)=\left\langle I^{\mathrm{Het}}(0) I^{\mathrm{Het}}(t) I^{\mathrm{Hom}}(\tau)\right\rangle_{c}=2 E^{2} \sum_{i j} a_{i}^{2} a_{j}^{2} \exp \left(-\Gamma_{i} \tau-\Gamma_{j}|t-\tau|\right)+C^{(2)}(t, \tau) .
\end{aligned}
$$

Denoting the time cumulants of $C_{M_{i}}=K_{r m}^{i}$, and taking $E^{2} \gg \bar{a}^{2}$ 


$$
\begin{aligned}
& K_{10}^{0}=-K_{10}^{\tau}=K_{01}^{0}=K_{01}^{t}=\frac{1}{2} K_{01}^{\tau}=\sum_{i} \bar{a}_{i}^{2} \Gamma_{i} /\left(\sum \bar{a}_{i}^{2}\right) \\
& K_{10}^{t}=K_{11}^{0}=0 \\
& K_{20}^{0}=\frac{1}{2} K_{20}^{t}=K_{20}^{\tau}=-K_{11}^{t}=-K_{11}^{\tau}=K_{02}^{0}=K_{02}^{t}=\frac{1}{2} K_{02}^{\tau}=\frac{1}{2} \sum_{i j} \bar{a}_{i}^{2} \bar{a}_{j}^{2}\left(\Gamma_{i}-\Gamma_{j}\right)^{2} /\left(\sum_{i, j} \bar{a}_{i}^{2} \bar{a}_{j}^{2}\right) .
\end{aligned}
$$

The time cumulants of these bispectra contain the same information that the cumulants of the regular spectrum do, namely, the average decay time and the mean-square range of decay times.

\section{SIGNAL CLIPPING AND THE HETERODYNE BISPECTRUM}

In measuring the correlation function $C^{(1)}(t)$, it is sometimes convenient to manipulate the recorded photocounts $I(t)$ before taking the cross products $I(t) I(t+\tau)$. It is usually asserted that certain standard manipulations, such as single clipping, scaling, and random scaling, change the signal-to-noise ratio without affecting the form of the spectrum. (There are known techniques, e.g., double clipping, which do distort the form of the spectrum.)

In this section, the effect of single clipped scaling on the heterodyne bispectrum is calculated. This clipping procedure is shown to change the form of the bispectrum in a novel way; the clipping procedure is shown to alter the symmetry of the correlation process so that the concentration-concentration correlation functions which contribute to the clipped heterodyne bispectrum are not the same as the concentration-concentration correlation functions which contribute to the full (unclipped) correlation functions. Specifically, it was assumed above that triple moments of the density such as $\left\langle a_{0} a_{t}^{*} a_{3}\right\rangle$ vanish on time averaging because the product is equally likely to be positive or negative. However, if the heterodyne bispectrum is measured and if one clips the signal at the average light level, one is removing from $\left\langle a_{0} a_{t}^{*} a_{\tau}\right\rangle$ all terms in which $a_{0}<0$, leaving only terms of positive sign, which clearly cannot cancel each other. The clipped heterodyne bispectrum thus depends in part on the odd powers of the density fluctuations, and therefore has a different time dependence than the full heterodyne bispectrum. It is interesting to note that single clipping also introduces terms depending on odd powers of $a_{0}$ into the regular heterodyne spectrum $C_{\text {fiet }}^{(i)}(\tau)$, so that single-clipped and unclipped heterodyne spectra also do not have the same time dependence, a fact sometimes not emphasized in the literature.

Here we calculate the difference between the clipped and unclipped cumulant spectra. This may usefully be done by introducing the antisymmetrized triple moment

$$
\rangle_{0} a_{0} a_{t}^{*} a_{\tau}\right\rangle=\left\langle\hat{a}_{0} a_{t} a_{\tau}^{*}\right\rangle,
$$

where $\hat{a}_{0}=a_{0}\left(a_{0}>0\right)$ and $\left.\left.\hat{a}_{0}=-a_{0} . \quad\left(a_{0}<0\right)\right\rangle_{0} a_{0} a_{t}^{*} a_{\tau}\right\rangle$ is the difference between the clipped and unclipped triple moments. The discussion is usefully phrased in terms of the three time transition matrix (matrix of conditional probabilities) $P_{t, \tau}\left(a_{1}, a_{2}, a_{9}\right)$ which gives the probability of observing amplitudes $a_{1}, a_{2}$, and $a_{3}$ at times $0, t$, and $\tau$, respectively. The symmetric correlation function is

$$
\left\langle a_{0} a_{t} a_{\tau}\right\rangle=\sum_{a_{1}, a_{2}, a_{3}=-\infty}^{\infty} a_{1} a_{2} a_{3} P_{t, \tau}\left(a_{1}, a_{2}, a_{3}\right),
$$

while the nonsymmetric function discussed here is

$$
\rangle_{0} a_{0} a_{t} a_{\tau}\right\rangle=\sum_{a_{2}, a_{3}=-\infty}^{\infty}\left(\sum_{a_{1}>0} a_{1} a_{2} a_{3} P_{t, \tau}\left(a_{1}, a_{2}, a_{3}\right)-\sum_{a_{1}<0} a_{1} a_{2} a_{3} P_{t, \tau}\left(a_{1}, a_{2}, a_{3}\right)\right) \text {. }
$$

To measure $\left.\rangle_{0} a_{0} a_{t} a_{\tau}\right\rangle$, the clipped variable $a_{0}^{\dagger}$ is introduced.

$$
a_{0}^{\dagger}=a, \quad a_{0}>0, \quad a_{0}^{\dagger}=0, \quad a_{0}<0 \text {. }
$$

Measurements of $a_{0}^{\dagger}$ may be implemented by post-detection digital manipulation of $a_{0}$. One has

$$
\left\langle a_{0} a_{t} a_{\tau}\right\rangle_{\mathrm{clipped}} \equiv\left\langle a_{0}^{\dagger} a_{t} a_{\tau}\right\rangle=\sum_{a_{2}, a_{3}=-\infty}^{\infty} \sum_{a_{1}>0}^{\infty} a_{1} a_{2} a_{3} P_{t, \tau}\left(a_{1}, a_{2}, a_{3}\right) \text {. }
$$

By breaking the sum on $a_{1}[\mathrm{Eq} .(4.2 a)]$ into its positive and negative parts, Eqs. (4.2) and (4.4) may be combined

$$
\rangle_{0} a_{0} a_{t} a_{\tau}\right\rangle=2\left\langle a_{0}^{\dagger} a_{t} a_{\tau}\right\rangle-\left\langle a_{0} a_{t} a_{\tau}\right\rangle \text {. }
$$

While Eq. (4.5) refers explicitly to the triple moment $a_{0} a_{t} a_{\tau}$, the derivation uses identities on sums of moments of the transition matrix, so Eq. (4.5) is valid for other moments of $a$.

$a_{0}^{\dagger}$ may be obtained by heterodyne detection of the scattered light. The heterodyne intensity is

$$
I^{\text {Het }}=E^{2}+2 E a_{0 R}+\left|a_{0}\right|^{2} ;
$$

clipping at $I^{\text {Het }}=\bar{I}^{\mathrm{Het}}$ is almost exactly equivalent to clipping at $a_{0 R}>0$. In the Appendix, the prescription of Eq. (4.3) is shown equivalent to clipping $a_{0}$ at $\operatorname{Re}\left(a_{0}\right)=\frac{1}{16} \bar{a}^{2} / E$. In a photon counting experiment, clip levels are necessarily integers, so in a real experiment there will necessarily be a systematic error in the clip level. In a large linear system this error will simply reduce the efficiency of data collection.

The effect of signal clipping of the sort indicated by Eq. (4.3) is here calculated first for the conventional hetero- 
dyne spectrum and then for the all-heterodyne bispectrum. A single solute is assumed. The clipped cumulant spectrum is

$$
\rangle_{0}(I(0)-\bar{I})(I(t)-\bar{I})\right\rangle=2\langle(I(0)-\bar{I})(I(t)-\bar{I})\rangle_{\mathrm{clipped}}-\langle I(0) I(t)\rangle_{c},
$$

where $I(0)$ is given by Eq. (4.6) and $\bar{I}=E^{2}+\bar{a}^{2}$. By direct calculation

$$
\langle(I(0)-\bar{I})(I(t)-\bar{I})\rangle=2 E^{2} \bar{a}^{2} e^{-\Gamma t}+\bar{a}^{t} e^{-2 \Gamma t},
$$

while for the clipped average

$$
\begin{aligned}
& \left\langle(I(0)-\bar{I}(I(t)-\bar{I})\rangle_{\text {c1 ldped }}\right. \\
& \quad=4 E^{2}\left\langle a_{0 R} a_{t R}\right\rangle_{C_{1}}+2 E\left\langle a_{0 R}\left(a_{t R}^{2}-a^{-2}\right)\right\rangle_{C_{1}}+2 E\left\langle a_{t R}\left(a_{0}^{2}-\bar{a}^{2}\right)\right\rangle_{C 1}+\left\langle\left(a_{0}^{2}-\bar{a}^{2}\right)\left(a_{t}^{2}-\bar{a}^{2}\right)\right\rangle_{C 1} .
\end{aligned}
$$

For $n$ even

$$
\left\langle a_{0 R}^{n}\right\rangle_{C 1}=\frac{1}{2}\left\langle a_{0 R}^{n}\right\rangle=\frac{1}{2} \int_{-\infty}^{\infty} d a_{0 R} a_{0 R}^{n} \frac{\exp \left(-a_{0 R}^{2} / \bar{a}^{2}\right)}{\sqrt{\pi} \bar{a}} .
$$

For $n$ odd, the lower limit on the integral becomes

$$
\left\langle a_{0 R}^{n}\right\rangle_{C 1}=\int_{0}^{\infty} d a_{0 R} a_{0 R}^{n} \frac{\exp \left(-a_{0 R}^{2} / a^{-2}\right)}{\sqrt{\pi} \bar{a}} .
$$

so

$$
\langle(I(0)-\bar{I})(I(t)-\bar{I})\rangle_{C 1}=E^{2} \bar{a}^{2} e^{-\Gamma t}+\frac{1}{2} E\left(\bar{a}^{3} / \sqrt{\pi}\right)\left(e^{-2 \Gamma t}+e^{-\Gamma t}\right)+\frac{1}{2} \bar{a}^{4} e^{-2 \Gamma t},
$$

and the antisymmetrized heterodyne intensity cumulant is

$$
\rangle_{0} I^{\text {Het }}(0) I^{\text {Het }}(t)\right\rangle_{c}=E\left(\bar{a}^{3} / \sqrt{\pi}\right)\left(e^{-2 \Gamma t}+e^{-\Gamma t}\right) .
$$

Equation (4.12) contains terms in $E^{1} \bar{a}^{3}$, which terms are not predicted by many analyses of the effects of signal clipping on the heterodyne spectrum. While these terms are weaker than the $E^{2} \bar{a}^{2}$ terms which dominate the heterodyne spectrum, they are only weaker by a factor $\bar{a} / E$, and therefore may contribute significantly to the clipped heterodyne spectrum even when $E^{2} \gg \bar{a}^{2}$. These terms arise because the clipping procedure of Eq. (5.3) treats $a_{0 R}$ $>0$ and $a_{0 R}<0$ in different ways, destroying the symmetry which causes $\left\langle a_{0 R}\left|a_{t}\right|^{2}\right\rangle$ to vanish. Consequently, in discussing how clipping affects a heterodyne spectrum, the spectrum itself must be recalculated, a calculation of the effect of clipping on the signal-to-noise ratio of the unclipped spectrum being insufficient.

On computing the antisymmetrized, all-heterodyne cumulant bispectrum, all terms involving even powers of $E$ and $\bar{a}$ cancel. Taking $E^{2} / \bar{a}^{2} \gg 1$,

$$
\rangle_{0} I^{\text {Het }}(0) I^{\text {Het }}(t) I^{\text {Het }}(\tau)\right\rangle_{c}=4 E^{3}\left(\bar{a}^{3} / \sqrt{\pi}\right)\left(e^{-\Gamma(t+\tau)}+e^{-\Gamma|t-\tau|}\right)+O\left(E \bar{a}^{5}\right),
$$

which is different from $\left\langle I^{\text {Het }}(0) I^{\text {Het }}(t) I^{\text {Het }}(\tau)\right\rangle_{c}\left[\mathrm{Eq}\right.$. (3.4)]. The time cumulants of (4.14) are $K_{10}=0, K_{01}=\Gamma, K_{20}$ $=\Gamma^{2}, K_{11}=0$, and $K_{02}=0$. The antisymmetrized bispectrum of a one-solute system tells as much about the system as the conventional spectrum does, but in a different way. Fitting Eq. (4.14) to the forms calculated in Sec. III would give erroneous results.

\section{SIGNAL-TO-NOISE RATIO IN A BISPECTRAL MEASUREMENT}

In this section, we show that bispectral analysis should work experimentally, in the sense that measurements of the spectrum and bispectrum should obtain similar signal-to-noise ratios. The demonstration has two parts: (a) the post-detection signal-to-noise ratio is shown to be well behaved, i.e., it increases as the square root of the duration of the measurement; (b) the pre-detection signal-to-background ratio of the homodyne spectrum and the homodyne bispectrum are shown to be nearly the same. If $C^{(1)}(t)$ and $C^{(2)}(t, \tau)$ can be measured equally accurately, they can be used to measure time cumulants to the same order in time, as was assumed in Secs. II-IV.

The moments and cumulants are obtained by time averaging, terms such as $\left\langle I_{0}\right\rangle\left\langle I_{t} I_{\tau}\right\rangle$ being computed as $\left\langle I_{u} I_{t} I_{\tau}\right\rangle$, where $u$ is a time much greater than any correlation time in the system. For the triple moment the integrated signal $\langle S\rangle$ is

$$
\begin{aligned}
\langle S\rangle \equiv & \int_{0}^{T} d s\left|a_{s}\right|^{2}\left|a_{s+t}\right|^{2}\left|a_{s+\tau}\right|^{2} \\
= & \int_{0}^{T} d s\left|a_{0} e^{-\Gamma s}+e^{-\Gamma s} \int_{0}^{s} e^{\Gamma \zeta_{i}} A\left(\zeta_{i}\right) d \zeta_{i}\right|^{2}\left|a_{0} e^{-\Gamma(s+t)}+e^{-\Gamma(s+t)} \int_{0}^{s+t} d \zeta_{i} e^{\Gamma \zeta_{i}} A\left(\zeta_{i}\right)\right|^{2} \mid a_{0} e^{-\Gamma(s+\tau)} \\
& \quad+\left.e^{-\Gamma(s+\tau)} \int_{0}^{s+\tau} d \zeta_{i} e^{\Gamma \zeta_{i}} A\left(\zeta_{i}\right)\right|^{2}
\end{aligned}
$$

where the index $i$ runs from 1 to 6 through the six terms. Rearranging terms 


$$
\langle S\rangle=\int_{0}^{T} d s \exp [-\Gamma(6 s+2 t+2 \tau)]\{a, A\},
$$

where $\{a, A\}$ is a polynomial in $a_{0}$ and $\int A(\zeta) \exp (\Gamma \zeta)$. A full expansion of $\{a, A\}$, which would be quite long, is not needed. From Eqs. (2.3) and (2.4), each term of $\{a, A\}$ depends on $s$ like $\exp (n \Gamma s), n=0,2,4,6$. If $n<6$, the integral on $s$ is $\int \exp (-a \Gamma s)=[\exp (-a \Gamma T)-1] /(-a \Gamma)$, which at large $T$ is independent of integrating time. The only terms of $\{a, A\}$ which contribute to $\langle S\rangle$ terms proportional to $T^{1}$ are from the terms containing all six factors of $A\left(\zeta_{1}\right)$, since these terms can be proportional to $\exp (+6 \Gamma s)$, so as to cancel the overall $s$ dependence of these terms of the integral (4.2). The sixfold integral over $\zeta_{i}$ may be done by using the Gaussian random nature of the $A\left(\zeta_{i}\right)$ to write

$$
\left\langle A\left(\zeta_{1}\right) A^{*}\left(\zeta_{2}\right) A\left(\zeta_{3}\right) A^{*}\left(\zeta_{4}\right) A\left(\zeta_{5}\right) A^{*}\left(\zeta_{8}\right)\right\rangle=\sum_{\substack{\{i, j, k\}=\{1,3,5\} \\\{m, n, p\}=\{2,4,6\}}}\left\langle A\left(\zeta_{i}\right) A^{*}\left(\zeta_{m}\right)\right\rangle\left\langle A\left(\zeta_{j}\right) A^{*}\left(\zeta_{n}\right)\right\rangle\left\langle A\left(\zeta_{k}\right) A^{*}\left(\zeta_{p}\right)\right\rangle,
$$

the sum being over all permutations of $\{1,3,5\}$ and $\{2,4,6\}$. By using Eqs. (2.3) and (2.4)

$$
\begin{aligned}
\langle s\rangle= & \int_{0}^{T} d s \exp [-\Gamma(6 s+2 t+2 \tau)] \bar{a}^{6}\left[\left(e^{2 \Gamma s}-1\right)\left(e^{2 \Gamma(s+t)}-1\right)\left(e^{2 \Gamma(s+\tau)}-1\right)+\left(e^{2 \Gamma s}-1\right)^{2}\left(e^{2 \Gamma(s+\tau)}-1\right)\right. \\
& \left.+\left(e^{2 \Gamma s}-1\right)^{2}\left(e^{2 \Gamma(s+t)}-1\right)+2\left(e^{2 \Gamma s}-1\right)^{2}\{\exp [2 \Gamma(s+\min (t, \tau))]-1\}+\left(e^{2 \Gamma s}-1\right)(\exp [2 \Gamma(s+\min (t, \tau))]-1)^{2}\right]
\end{aligned}
$$

or

$$
\langle S\rangle=T \bar{a}^{6}\left\{1+e^{-2 \Gamma t}+e^{-2 \Gamma \tau}+e^{-2 \Gamma|t-\tau|}+2 \exp [-2 \Gamma \max (t, \tau)]\right\}+O\left(T^{0}\right) .
$$

Equations (5.1)-(5.5) are also sufficient for calculating the cumulant $\langle S\rangle_{c} .\left\langle I_{0} I_{t}\right\rangle\left\langle I_{\tau}\right\rangle$ is

$$
\int d s\left\langle\left|a_{s}\right|^{2}\left|a_{s+t}\right|^{2}\left|a_{s+u}\right|^{2}\right\rangle=T \bar{a}^{8}\left(1+e^{-2 \Gamma t}+e^{-2 \Gamma u}+e^{2 \Gamma(u-t)}+2 e^{-2 \Gamma u}\right)+O\left(T^{0}\right) .
$$

and similarly for $\left\langle I_{0} I_{\tau}\right\rangle\left\langle I_{t}\right\rangle$ and $\left\langle I_{0}\right\rangle\left\langle I_{t} I_{\tau}\right\rangle$. Terms in $\exp (-2 \Gamma u)$ vanish, so that

$$
\langle S\rangle_{c}=2 \bar{a}^{6} T \exp [-2 \Gamma \max (t, \tau)] \text {. }
$$

The integrated signal therefore grows linearly in time. For a practical experiment one also needs to show that the noise (the fluctuations in $\langle S\rangle_{c}$ ) grow more slowly than the integrated signal. The noise $\langle N\rangle$ may be calculated as

$$
\langle N\rangle=\left(\left\langle S^{2}\right\rangle-\langle S\rangle^{2}\right)^{1 / 2}
$$

The mean-square signal is

$$
\begin{aligned}
\left\langle s^{2}\right\rangle= & \int_{0}^{T} \int_{0}^{T} d r d s\left(\left|a_{r}\right|^{2}\left|a_{r+t}\right|^{2}\left|a_{r+r}\right|^{2}-\left|a_{r+u}\right|^{2}\left|a_{r+t}\right|^{2}\left|a_{r+\tau}\right|^{2}-\left|a_{r}\right|^{2}\left|a_{r+u}\right|^{2}\left|a_{r+\tau}\right|^{2}\right. \\
& \left.-\left|a_{r}\right|^{2}\left|a_{r+t}\right|^{2}\left|a_{r+u}\right|^{2}+2\left|a_{r}\right|^{2}\left|a_{r+u}\right|^{2}\left|a_{r+2 u}\right|^{2}\right)\left(\left|a_{s}\right|^{2}\left|a_{s+t}\right|^{2}\left|a_{s+\tau}\right|^{2}-\left|a_{s+u}\right|^{2}\left|a_{s+t}\right|^{2}\left|a_{s+r}\right|^{2}\right. \\
& \left.-\left|a_{s}\right|^{2}\left|a_{s+u}\right|^{2}\left|a_{s+r}\right|^{2}-\left|a_{s}\right|^{2}\left|a_{s+t}\right|^{2}\left|a_{s+u}\right|^{2}+2\left|a_{s}\right|^{2}\left|a_{s+u}\right|^{2}\left|a_{s+2 u}\right|^{2}\right) .
\end{aligned}
$$

which may be rewritten

$$
\left\langle s^{2}\right\rangle=\iint d r d s \exp [-\Gamma(6 r+6 s)]\{a, A\}^{\prime} .
$$

The only terms of $\{a, A\}^{\prime}$ whose integrals grow faster than $T^{0}$ at long times are those containing twelve factors of $A\left(\zeta_{1}\right)$. The average over the prodact of the $A\left(\zeta_{i}\right)$ factors into a series of products of averages over pairs $\left\langle A\left(\zeta_{1}\right) A^{*}\left(\zeta_{j}\right)\right\rangle$. The products of averages may be divided into the cross terms between $a_{r}$ and $a_{s}$, which all contain double integrals involving both $r$ and $s$ such as

$$
I_{1}=\left\langle\int_{0}^{r} \int_{0}^{s} d \zeta_{i} d \zeta_{2} \exp \left[\Gamma\left(\zeta_{1}+\zeta_{2}\right)\right] A\left(\zeta_{1}\right) A^{*}\left(\zeta_{2}\right)\right\rangle
$$

and the non cross terms, in which limits involving both $r$ and $s$ do not appear in the same double integral. The non cross terms are the same as those appearing in $\langle S\rangle^{2}$ and cancel the $-\langle S\rangle^{2}$ of Eq. (5.8); the cross terms contribute to $\langle N\rangle$. A typical cross term is

$$
\begin{aligned}
I=\int_{0}^{T} \int_{0}^{T} d r d s \exp [-\Gamma(6 r+6 s+4 t+4 \tau)]\left|\int_{0}^{r} \int_{0}^{s} \exp \left[\Gamma\left(\zeta_{1}+\zeta_{2}\right)\right] A\left(\zeta_{1}\right) A^{*}\left(\zeta_{2}\right)\right|^{2} \bar{a}^{\mathrm{B}} \\
\quad \times\left(e^{2 \Gamma(r+t)}-1\right)\left(e^{2 \Gamma(r+\tau)}-1\right)\left(e^{2 \Gamma(s+t)}-1\right)\left(e^{2 \Gamma(s+r)}-1\right) .
\end{aligned}
$$

The final integrals over $\zeta$ are $[\exp (2 \Gamma \min (r, s))-1]^{2}$; choosing $r<s$, the only term of (5.11) which does not decay exponentially with $r$ and $s$ is 
$\int_{0}^{T} \int_{0}^{T} d s d r e^{-2 \Gamma(s-r)} \bar{a}^{-12}=2 \bar{a}^{12}\left(T+\frac{\left(e^{-2 \Gamma T}-1\right)}{2 \Gamma}\right)$,

the integral being done by a change of variables: $r+s$ $=\omega, r-s=u$. The cross terms in $\left\langle s^{2}\right\rangle$ thus depend on $T$ no more strongly than $T^{1}$, so that

$$
N=\left(\left\langle S^{2}\right\rangle-\langle S\rangle^{2}\right)^{1 / 2} \propto T^{1 / 2} .
$$

Since the total integrated signal increases linearly with integration time $T$, the signal-to-noise ratio increases as $T^{+1 / 2}$, as required for a well-behaved experiment.

One also wants to know how favorable the initial signalto-noise ratio is. Even though $S / N$ is well behaved, if the pre-detection signal-to-noise ratio were poor, a successful experiment could require excessive amounts of integrating time. Comparing the signal and total intensities at $t=\tau=0$, one finds for the initial signal-tobackground ratios: (i) one component, all homodyne,

$$
\frac{C^{(2)}(0,0)}{I(0,0)}=\frac{1}{3}
$$

(ii) $N$ components, all homodyne,

$$
\frac{C^{(2)}(0,0)}{I(0,0)}=\frac{1}{3}
$$

(iii) $N$ components, all heterodyne,

$$
\frac{C_{\mathrm{Het}}^{(2)}(0,0)}{I(0,0)}=\frac{6\left(\sum \bar{a}_{i}^{2}\right)^{2}}{E^{4}} ;
$$

(iv) $N$ components, mixed,

$$
\frac{C_{\mu 0}^{(2)}(0,0)}{I(0,0)}=\frac{C_{\mu_{t}}^{(2)}(0,0)}{I(0,0)}=\frac{C_{\mu_{t}}^{(2)}(0,0)}{I(0,0)}=\frac{2 \sum \bar{a}_{i}^{2}}{E^{2}} \text {. }
$$

The all-homodyne cumulant bispectra amount to $\frac{1}{3}$ of the detected intensity. By comparison the all-homodyne cumulant spectrum $C^{(1)}(t)$ is $\frac{1}{2}$ of the total intensity, so the predetection signal-to-noise ratio of $C^{(2)}(t, \tau)$ is not substantially worse than that of $C^{(1)}(t)$. The mixed and all-heterodyne bispectra are weaker than their backgrounds by factors of $\sum \bar{a}_{i}^{2} / E^{2}$ and $\left(\sum \bar{a}_{i}^{2}\right)^{2} / E^{4}$. The former is the same as the signal-to-background ratio in a normal heterodyne spectrum, while the latter is substantially worse. Careful precorrelation background subtraction would probably be required for observing $C_{\mathrm{Het}}^{(2)}(t, \tau)$; fortunately, $C_{\mathrm{Het}}^{(2)}(t, \tau)$ is substantially less interesting than $C^{(2)}(t, \tau)$ is.

\section{EXPERIMENTAL ASPECTS}

We find that the bispectrum may give considerably more information about a system than the conventional spectrum does. The bispectrum is not, however, considerably harder to measure than the regular spectrum. A digital bicorrelator designed to determine $C^{(2)}(t, \tau)$ need not be substantially more complicated than a conventional autocorrelator.

A conventional digital correlator works by dividing time into intervals, counting the number of photons received in each interval and recording these numbers by loading them sequentially into a shift register. The correlation function $\langle I(s) I(s+\tau)\rangle$ is calculated by multiplying together the number of photocounts received at times $s$ and $s+\tau$, storing the product separately for each value of $\tau$, and time averaging by repeating the process for a series of values of $s$. In most designs, $s+\tau$ is the current instant of time, the number of photocounts received at the earlier time $s$ being obtained from the shift register. A similar design gives one a bicorrelator. To measure $C^{(2)}(t, \tau)$, one multiplies the number of counts received at time $(s+\tau)$ by the number of counts received at each of the two previous times $s+t$ and $s$. This requires two multiplications; values of $I(s+t)$ and $I(s)$ may be found either by examining two positions in a single shift register or by using two shift registers, one for $I(s)$ and one for $I(s+t)$. The use of two shift registers ought to be preferred, in that a two-shift register design permits cross correlation of three different signals, while the single-shift register design can only cross correlate two signals.

Modern digital correlators can compute the correlation function simultaneously at as many as 400 points, thus having 400 output channels. A similarly designed bicorrelator has two time variables, and thus would need $(400)^{2}$, or 16000 , channels. With some care, the number of channels in a well-designed bicorrelator can be kept under 500 without significant loss of accuracy.

The main reason that one does not need $\sim 10^{4}$ channels for a bicorrelator is that increasing the number of channels does not give a proportionate increase in the measurement accuracy. If one increases the number of channels in the first few decay times, one reduces the number of photons received per channel time span, which reduces the signal-to-noise ratio of each channel. On the other hand, increasing the number of channels by increasing the number of decay times over which the correlation function is measured is not extremely effective because at long decay times the correlation function is nearly constant. By spacing channels unevenly in time, accurate measurements of the first and second time cumulant for a paucidisperse system are possible with a correlator having as few as 19 or 32 channels. ${ }^{8}$ A correspondingly built bicorrelator would have 4001000 channels. Since multiplication is commutative $C^{(2)}(t, \tau)=C^{(2)}(\tau, t)$; by requiring $t<\tau$, the number of channels is reduced by a further factor of 2 . For the experiments discussed in this paper, a digital bicorrelator would require no more than 500 channels.

Thus far, we have considered only bispectra measured in time domain, the Fourier transform from $C^{(2)}(t, \tau)$ to $S^{(2)}\left(\omega_{1}, \omega_{2}\right)$ playing no significant part in the results. Brillinger ${ }^{1}$ demonstrates that polyspectra may also be measured in frequency domain by a complex demodulation technique. Namely, one takes real Fourier transforms $U$ and $U^{H}$ of the signal $I(t)$

$$
\begin{aligned}
& U(\omega)=T^{-1} \int_{0}^{T} d t I(t) \cos (\omega t) \\
& U^{H}(\omega)=T^{-1} \int_{0}^{T} d t I(t) \sin (\omega t),
\end{aligned}
$$

as may be done with a bank of phase-sensitive frequency filters, and then uses $U(\omega)$ and $U^{H}(\omega)$ to compute poly spectra. The use of smoothing factors to improve the convergence of Eqs. (6.1) is treated by Brillinger. Frequency domain measurements have several advantages 
over time domain measurements. The $U(\omega)$ and $U^{H}(\omega)$ may be used to computer polyspectra of arbitrary order, while $C^{(2)}(t, \tau)$ only gives the bispectrum. Furthermore, digital computation of $C^{(2)}(t, \tau)$ is limited to rather lower frequencies than phase-sensitive filtering is. On the other hand, theoretical treatments of diffusion are more readily made in time domain.

\section{DISCUSSION}

Experimental applications of bispectral analysis are quite rare, so little comparison between our results and the literature is possible. Kim, Powers, and coworkers ${ }^{2}$ have discussed bispectral analysis of nonlinear systems, applying their results to an experimental study of density and potential fluctuations in a plasma. Hasselman et al. ${ }^{3}$ report experimental data on the bispectra of ocean waves. Sato et al. " have used a bispectral analyzer in a laser doppler system, studying the forced response of aerosol particles to nonsinusoidal sound waves of fixed bispectral characteristics. The noise in their experiment was largely an additive Gaussian background; because Gaussian signals have a zero bispectrum, bispectral analysis was successfully used to obtain a bispectral signal in the presence of strong background noise.

In a previous paper, ${ }^{5}$ we discussed how light scattering studies of a third order intensity correlation function can be used to measure the elsewise experimentally inac cessible triple dynamic structure factor $S^{(3)}(\mathbf{k}, q, t, \tau)$ of a bulk system. In that experiment, it was proposed to illuminate the sample with two laser beams and study cross correlations in intensity fluctuations at three distinct coherence areas (as opposed to the single coherence area considered here), the detectors necessarily being operated in the heterodyne mode. The triple crosscorrelation function $C^{(2)}(t, \tau)=\left\langle I_{1}(0) I_{2}(t) I_{3}(\tau)\right\rangle$ is deter mined by $S^{(3)}\left(\mathbf{k}, \mathrm{q}, t, t^{\prime}\right)$. The bispectral aspects of this experiment are secondary to the use of phase-sensitive detection, which serves to suppress terms dependent on the pair correlation function $S^{(2)}(k)$.

Our main interesting conclusion is that bispectral analysis can be usefully applied in light scattering spectroscopy, especially in the study of complex systems. In particular, the all-homodyne bispectrum can effectively distinguish between bidisperse and polydisperse systems. All-heterodyne and mixed bispectra are less useful than the all-homodyne bispectrum.

We illustrate the utility of bispectral analysis by considering light scattering from a tridisperse system with amplitudes $a_{1}=a_{2}=a_{3}$ and decay times $\Gamma, 2 \Gamma$, and $3 \Gamma$, respectively. The spectrum of this system would show an average decay time of $2 \Gamma$ and a variance $\left(\sqrt{K}_{2} / K_{1}\right)$ $=0.41$. A bidisperse system with $a_{1}=a_{2}$ and $\Gamma_{1}=1.18 \Gamma$, $\Gamma_{2}=2.82 \Gamma$ would yield the same values for the average diffusion coefficient and variance. Measurement of the spectrum to the usually attainable level of accuracy ther efore cannot distinguish between the model tridisperse system and a matching bidisperse system. In order to distinguish between the two and three exponential systems by measuring $C^{(1)}(t)$, one would need to obtain an ac- curate numerical value for $K_{4}$, which is usually impossible.

A measurement of the bispectrum would immediately distinguish the tridisperse system from its bidisperse model. The bispectral time cumulants for the tridisperse system would be $K_{10}=0, K_{01}=2 \Gamma$

$$
K_{20}=\frac{24}{27} \Gamma^{2}, K_{11}=-\frac{16}{27} \Gamma^{2} \text {, and } K_{02}=\frac{42}{2} \Gamma^{2}
$$

In a bisdisperse system, $K_{20}+2 K_{11}=0$; in this system $K_{20}+2 K_{11}=\frac{-8}{27} \Gamma^{2}$ which corresponds to a variance $\left(K_{20}\right.$ $\left.+2 K_{11}\right)^{1 / 2} / K_{01}=0.27$. Variances of this magnitude may readily be measured [at least in $C^{(1)}(t)$, whose signalto-noise ratio is the same as that of $\left.C^{(2)}(t, \tau)\right]$, so a bispectral study of the tridisperse system will readily serve to reject the hypothesis that the system is only bidisperse.

We emphasize the difference between this result, showing the utility of bispectral analysis and the result which is obtained if one ignores the noise in the experiment. With a (mythical) noise-free measurement of $C^{(1)}(t)$, one could fit the spectrum exactly to a sum of $n$ exponentials, determining not only how many exponentials are present but also their amplitudes and decay times; measuring $C^{(2)}(t, \tau)$ would be entirely redundant. In a real experiment on a moderately polydisperse system, $C^{(1)}(t)$ gives only limited information on the distribution of exponentials; with the same signal-to-noise ratio, $C^{(2)}(t$, $\tau)$ gives more information than $C^{(1)}(t)$ does.

Being able to distinguish between bidisperse and polydisperse suspensions is of specific interest in the problem of protein denaturation. Light scattering has been used to observe the expansion of lysozyme and other protein molecules during thermal denaturation ${ }^{9}$; the results are consistent with a two-step model for protein denaturation but do not entirely exclude a more complicated process. In favorable system, bispectral light scattering should be able to distinguish two-step and more complicated denaturation processes.

In nonideal solutions, interactions between solute molecules may give rise to nonexponential decays of concentration fluctuations. ${ }^{10}$ A cumulant analysis of the spectrum $^{11}$ is clearly suitable for slightly nonexponential decays. For strongly nonexponential behavior, an interpretation of the spectrum is sometimes made in terms of two exponentials, "12 "fast" and "slow," even though comparison with mode-coupling theories suggests that a long-time power law decay might be expected. The results of this paper do not indicate precisely what the bispectrum might reveal about the dif fusion of interacting species. In an interacting system, each species has a complex relaxation; here we have assumed that each species has its own exponential relaxation.

Our results indicate that in bispectral analysis, homodyne detection of the scattered light is to be preferred to heterodyne detection. The signal-to-noise ratios obtained for $C_{\mathrm{Hom}}^{(2)}(t, \tau)$ and $C^{(1)}(t)$ are predicted to be the same. For suspensions of noninteracting Brownian particles, bispectral analysis following heterodyne de tection gives the same sort of information that regular 
spectral analysis does, with an inferior signal-to-noise ratio. The use of signal clipping in bispectral analysis of heterodyne-detected light is predicted to lead to experimental difficulties, in that the clipped and unclipped bispectra depend on different concentration-concentration correlation functions. As is sometimes not pointed out, this final effect also occurs with the regular heterodyne spectrum.

\section{APPENDIX: RELATION BETWEEN CLIP LEVELS OF IHet AND $a_{R}$}

To measure the antisymmetric correlation function \rangle$\left._{0} a_{0} a_{t} a_{\tau}\right\rangle$, one needs to separate the signals with $a_{0 R}>0$, $a_{0 R}$ being the real part of $a_{0}$. Section IV proposed to do this by clipping the heterodyne intensity at $I^{\text {Het }}=\left\langle I^{\text {Het }}\right\rangle$; that is, one clips at $t=0$ by considering only signals which satisfy

$$
E^{2}+2 E a_{R 0}+\left|a_{0}\right|^{2}>E^{2}+\bar{a}^{2} .
$$

In the limit $E / a_{0}-\infty$ (A1) is only satisfied for $a_{R 0}>0$.

Here we examine more generally the relation between clipping $I^{\mathrm{Hot}}$ and actually clipping $a_{R 0}$, by calculating the average value of which $a_{R 0}$ attains when (A1) obtains as an equality. This is, we consider the average value of $a_{R 0}$ satisfying

$$
2 E a_{R 0}+\left|a_{0}\right|^{2}-\bar{a}^{2}=0 .
$$

Since $\left|a_{0}\right|^{2}=a_{R 0}^{2}+a_{T 0}^{2}$, and $\bar{a}_{R 0}^{2}=\bar{a}_{J 0}^{2}=\frac{1}{2} \bar{a}^{2}, \mathrm{Eq}$. (A2) is equivalent to

$$
\left\langle a_{R 0}\right\rangle \cong\left\langle-E_{ \pm \frac{1}{2}}\left[4 E^{2}-\left(a_{I 0}^{2}-\bar{a}^{2}\right)\right]^{1 / 2}\right\rangle,
$$

For $E^{2} \gg a^{2}$, this becomes

$$
\left\langle a_{R 0}\right\rangle \cong\left\langle E\left(\bar{a}^{2}-a_{I}^{2}\right) / 8 E^{2}\right\rangle=\bar{a}^{2} / 16 E,
$$

which is substantially smaller than $\bar{a}$.

${ }^{1}$ D. R. Brillinger, Ann. Math. Stat. 36, 1351 (1965).

${ }^{2}$ Y. C. Kim and E. J. Powers, Phys. Fluids 21, 1452 (1978). ${ }^{3} \mathrm{~K}$. Hasselman, W. Munk, and G. MacDonald, in Time Series Analysis, edited by M. Rosenblatt (Wiley, New York, 1963), pp. $125-139$.

${ }^{4}$ T. Sato, T. Kishimoto, and K. Sasaki, Appl. Opt. 17, 667 (1978).

${ }^{5}$ G. D. J. Phillies, Mol. Phys. 32, 1695 (1976).

${ }^{6}$ G. E. Uhlenbeck and L. S. Ornstein, Phys. Rev. 36, 823 (1930).

${ }^{7}$ D. E. Koppel, J. Chem. Phys. 57, 4814 (1972).

${ }^{8}$ G. D. J. Phillies, G. B. Benedek, and N. M. Mazer, J. Chem. Phys. 65, 1883 (1976); P. A. Doherty and G. B. Benedek, ibid. 61, 5426 (1975).

${ }^{9}$ D. F. Nicoli and G. B. Benedek, Biopolymers 15, 2421 (1976).

${ }^{10} \mathrm{~W}$. Hess and R. Klein, Physica 85A, 509 (1976).

${ }^{11}$ B. J. Ackerson, J. Chem. Phys. 64, 242 (1976).

${ }^{12}$ T. Nose and B. Chu, Macromolecules 12, 590 (1974). 